\title{
What is the Burden of Antimicrobial Resistance Genes in Selected Ready-to-Eat Foods?
}

Area of research interest: Antimicrobial resistance

Study duration: 2019-02-01

Project status: Completed

Project code: FS301050

Conducted by: FERA

Date published: 17 September 2021

DOI: https://doi.org/10.46756/sci.fsa.bsv485

\section{Background}

This study was designed to get a broad estimate of the presence and the types of antimicrobial resistance genes across 52 simple ready-to-eat foods. It was also carried out to understand the benefits and drawbacks of using metagenomic sequencing, a fairly new technology, to study AMR genes.

An antimicrobial is any substance that kills or inhibits the growth of microorganisms. It includes antibiotics which are used to treat bacterial infections in both humans and animals. Given the relevant selective pressures, the bacteria itself can change and ?nd ways to survive the effects of an antimicrobials. This results in the bacteria becoming resistant to the 'killing' effects of antimicrobials and is known as 'antimicrobial resistance'.

The more we use antimicrobials and antibiotics and the way that we use them can increase the chance that bacteria will become resistant to antimicrobials. This is important as it can lead to infections that become more difficult to treat with drugs and poses a risk to the public health. T

Addressing AMR is a national strategic priority for the UK Government which has led to the development of a new 20-year Vision for AMR and the 5-year National Action Plan (NAP), which runs until 2024. The NAP lays out how the UK will address the AMR challenge and takes a 'OneHealth' approach which spans people, animals, agriculture, food and the environment. The NAP includes a specific section on the importance of better food safety to limit the contamination of foods and spread of AMR. This section emphasises the need to strengthen the evidence base for AMR and food safety through research, surveillance and promoting good practice across the food chain.

The FSA is playing its part by continuing to fill evidence gaps on the role that food plays in AMR through the commissioning of research and surveillance. We are also promoting and improving UK food hygiene ('4Cs' messages) across the food chain that will help reduce exposure to AMR bacteria.

\section{Research approach}

In total, 256 samples across 52 different food types were sampled by the researchers, and these included 33 type of produce, 17 types of dairy, and two types of cooked meat. The number of 
samples of each type of food was based on their consumption in the average diet.

Bacterial DNA was extracted and submitted for metagenomic sequencing. The researchers then used online software and databases to identify AMR genes in each sample.

\section{Results}

Certain AMR genes such as efflux pumps were estimated by the researchers to be present in most diets. Genes associated with colistin and methicillin resistance were rarely found in the foods tested. More than 50 different types of fluoroquinolone resistance genes were found in various types of produce.

The study, however, did not analyse whether these genes were actively making the bacteria resistant to these antibiotics. Further work is necessary to understand whether the genes identified were working.

Some metagenomic sequencing technologies were found to work better than others - for instance, data generated using PromethION sequencing was poor, but in future this methodology can be improved, as the data generated is very valuable.

The study also found that it was more efficient to extract bacterial DNA from fruit like apples that could be rinsed, rather than foods such as milk. These are valuable insights that can be used in future projects when extracting and sequencing bacterial DNA from food.

\section{Report}

PDF

View What is the Burden of Antimicrobial Resistance Genes in Selected Ready-to-Eat Foods report as PDF(Open in a new window) (4.04 MB)

\section{Supporting data}

What is the burden of AMR genes in selected ready to eat foods? appendices published on data.food.gov. 